\title{
Tarefas e justificativas de professores em ambientes virtuais colaborativos de geometria dinâmica ${ }^{12}$
}

\author{
Tasks and teachers' justifications in virtual, collaborative, dynamic geometry \\ environments
}

Arthur B. Powell

Vinícius Pazuch ${ }^{4}$

\begin{abstract}
Resumo
Neste artigo reflete-se sobre justificativas geométricas elaboradas por professores que ensinam Matemática, usando relações e propriedades, e como eles resolvem uma tarefa sobre quadriláteros e suas bissetrizes no ambiente Virtual Math Teams with GeoGebra (VMTwG). Os professores são participantes do projeto de formação continuada com tecnologias digitais, trabalham em pequenas equipes colaborativamente para construir figuras geométricas e resolver problemas geométricos. Dada uma sequência de tarefas elaborada para discussão e resolução em um ambiente virtual colaborativo, analisa-se como três professores colaborativa e discursivamente construíram justificativas teóricas com base em suas próprias conjecturas e percepções de relações entre objetos geométricos, sem o uso de medidas. Essas justificativas teóricas e as condições em que elas foram realizadas geram implicações para a Educação Matemática de professores e para a prática docente deles.
\end{abstract}

Palavras-chave: relações geométricas, justificativas teóricas, tecnologias digitais, geometria dinâmica, formação inicial e continuada de professores

\begin{abstract}
In this article, we reflect on how mathematics teachers build geometric justifications based on geometric relations and properties as they solve a task about quadrilaterals and their angle bisectors, all within the online environment, Virtual Math Teams with GeoGebra (VMTwG). The teachers, participants in a continuing education project with digital technologies, work collaboratively in small teams to construct geometric figures and solve geometric problems. Given a sequence of tasks designed for discussion and resolution within a collaborative online environment, we analyze how three teachers collaboratively and discursively construct
\end{abstract}

\footnotetext{
${ }^{1}$ Este trabalho é baseado em uma pesquisa apoiada pela NSF, do programa DRK-12, sob a bolsa DRL-1118888. As conclusões e as opiniões relatadas aqui são de responsabilidade do autor e não refletem necessariamente a opinião da NSF.

${ }^{2}$ Uma versão preliminar e parcial do trabalho foi apresentada no VI SIPEM - Seminário Internacional de Pesquisa em Educação Matemática.

${ }^{3}$ Doutor em Educação Matemática pela Rutgers University. Professor da Rutgers University, EUA. Email: powellab@andromeda.rutgers.edu

${ }^{4}$ Doutor em Ensino de Ciências e Matemática, pela Universidade Luterana do Brasil, com estágio de Doutorado Sanduíche na Rutgers University (Processo: PDSE-6101/13-5), Pós-Doutorando na Universidade Federal do ABC, Brasil. Email: viniuch@ hotmail.com
} 
http://periodicos.sbu.unicamp.br/ojs/index.php/zetetike

theoretical justifications based on their own conjectures and perceptions of relations among geometric objects, without the use of measurement. These theoretical justifications and the conditions in which it manifested generate implications for mathematics teacher education and for teachers' classroom practice.

Keywords: geometric relations, theoretical justifications, digital technologies, dynamic geometry, pre- and inservice teacher education

\section{Introdução}

Tarefas e justificativas são alguns dos materiais que colocam a Matemática em movimento. Na Matemática, hoje em dia, a construção, a visualização e a manipulação de objetos geométricos, dependências e outras relações são possibilidades propiciadas pelo uso de Softwares de Geometria Dinâmica (SGD). Entre os usuários dos SGD, sejam estudantes ou professores, tais possibilidades apoiam determinadas ações corporais e cognitivas, explorações empíricas e justificativas teóricas (Christou, Mousoulides, Pittalis, \& PittaPantazi, 2004). Embora a motivação para essas ações também seja considerada inerente aos SGD, a ação anterior é imediata, enquanto a última, para ser realizada, exige disposição dos usuários tanto para a justificativa como para a intervenção pedagógica. Pedagogicamente transições de explorações empíricas para justificativas teóricas dependem de tarefas cuidadosamente elaboradas, de orientação do professor e de um "clima" na sala de aula que propiciem conjecturas e justificativas teóricas (Arzarello, Olivero, Paola \& Robutti, 2002; Hölzl, 2001; Öner, 2008). Um ambiente de sala de aula, incorporado a um ambiente colaborativo de aprendizagem apoiado por computador $\left(\mathrm{CSCL}^{5}\right.$ ) (Öner, 2008; Silverman, 2011), pode sustentar conjecturas e justificativas. Assim, usuários (sejam professores ou estudantes) necessitam de experiências nesses ambientes de aprendizagem.

Em tal cenário, questionamos: como podemos entender o movimento intrínseco de professores desde a exploração até as justificativas teóricas? Promover este movimento pode permitir que pesquisadores em Educação Matemática e educadores percebam o potencial do uso de SGD. Além disso, este movimento pode contribuir para que visualizem as potencialidades do uso de ambientes CSCL para melhorar a aprendizagem em geometria, envolvendo professores no desenvolvimento de ideias matemáticas, por meio da colaboração online e do trabalho colaborativo (Alagic \& Alagic, 2013).

Neste artigo, o nosso principal objetivo é indicar como determinadas tarefas juntamente com um SGD incorporado a um ambiente de CSCL podem promover o movimento intrínseco de professores da Educação Básica desde a exploração até o processo de conjecturas e de justificativa teórica. Consideramos que essa discussão é importante no âmbito tanto da formação inicial como da formação continuada, pois professores podem, posteriormente, promover investigações dessa natureza com seus estudantes em sala de aula.

\footnotetext{
${ }^{5}$ A sigla CSCL vem do inglês: computer supported collaborative learning. Para uma perspectiva histórica do CSCL, veja Stahl, Koschmann e Suthers (2008).
}

Zetetiké, Campinas, SP, v.24, n.2, maio/ago.2016, p.191-207

ISSN 2176-1744 
http://periodicos.sbu.unicamp.br/ojs/index.php/zetetike

\section{Literatura relacionada}

Dentre as diversas pesquisas que investigam a formação inicial ou continuada de professores de Matemática e o uso de SGD, encontramos duas que discutem a forma como professores resolvem uma tarefa vinculada à exploração de bissetrizes de um paralelogramo (Amaral, 2011; Christou et al., 2004). A tarefa consiste em construir um paralelogramo $\mathrm{ABCD}$; traçar as bissetrizes dos ângulos internos desse paralelogramo; questionar e discutir qual figura geométrica é formada pelas quatro bissetrizes; arrastar os pontos A, B, C ou D; questionar quais as condições necessárias para que o quadrilátero EFGH seja um quadrado; e questionar qual quadrilátero é obtido quando são traçadas as bissetrizes do quadrilátero EFGH.

Em respeito a essa tarefa, Christou et. al (2004) apresentam possibilidades de contribuição do SGD na elaboração de provas em geometria por meio de investigação e de experimentação. Nessa pesquisa, três professores do Ensino Fundamental trabalharam individualmente na resolução de problemas geométricos. Os pesquisadores apontam que o SGD ofereceu para esses professores a possibilidade de construir de forma apropriada, de explorar mudanças de propriedades das configurações geométricas distintas e de resolver o problema por meio do uso de medidas. Com a tarefa, segundo os pesquisadores, o uso das ferramentas de medida mobilizou os professores do empírico para justificativas formais, esclarecendo que a prova não é separada da ação. A ação é expressa pela construção de formas e medidas, enquanto a prova é expressa por dependências entre propriedades.

Usando a mesma tarefa em um curso online com o uso de videoconferências e do software Geometricks, Amaral (2011) investigou argumentações matemáticas colaborativas de professores relacionadas à resolução da tarefa. As argumentações produzidas aconteceram por meio de diálogos entre os dois organizadores do curso e os professores. Os resultados apontam que os professores usaram medidas para provar conjecturas como, por exemplo, se uma figura geométrica plana é quadrado ou retângulo. A autora conclui que os professores sabiam usar as propriedades matemáticas na resolução das atividades, mas não conseguiam encontrar argumentos matemáticos para justificá-las.

A partir dessas duas pesquisas, compreendemos que as justificativas geométricas usadas pelos professores foram baseadas no uso de medidas. No entanto, na Geometria Euclidiana sintética (sem coordenadas), as proposições devem ser estabelecidas teoricamente com base nas propriedades dos objetos geométricos e das relações que existem entre eles. As conjecturas só podem ser confirmadas com o uso das relações e não das medidas. Isto porque as medidas são imprecisas e dependem das ferramentas usadas para medição. Em contraste, usando a mesma tarefa, apresentamos como professores, colaborativamente, estabelecem relações geométricas para elaborar justificativas teóricas. As interações entre eles ocorreram no ambiente VMTwG. Tratamos teoricamente das Tecnologias Digitais (TD) como espaço de reflexão, argumentação e construção de relações geométricas, de forma colaborativa. 
http://periodicos.sbu.unicamp.br/ojs/index.php/zetetike

\section{Fundamentação teórica}

Ao usarmos TD para construir ideias matemáticas, possivelmente, enfrentamos desafios. Powell (2014) apresenta dois deles. O primeiro é que os avanços das TD requerem que pesquisadores e educadores investiguem as potencialidades dessas para a Educação. Especificamente, o autor pontua que, em Educação Matemática, há poucos estudos que abordam a colaboração como uma das potencialidades fundamentais da Web 2.0 e, por consequência, que há necessidade de pesquisas com professores que ensinam Matemática na Educação Básica, que investiguem o que a TD pode ajudar aprendizes na construção de ideias matemáticas e como ela contribui para isso.

O segundo desafio está relacionado à necessidade de colaboração para encontrar soluções relativas à elaboração de projetos, à criação de novos produtos, mídias e serviços para melhorar a vida do planeta e de seus habitantes. Na Educação Matemática, a elaboração de tarefas pode moldar como grupos de indivíduos percebem e constroem relações entre os objetos matemáticos e, por conseguinte, suas construções de conhecimentos matemáticos.

Tarefas em um ambiente de CSCL requerem caraterísticas próprias que são diferentes daquelas direcionadas para ambientes tradicionais de salas de aula. As deferências são que o professor não está presente contemporaneamente em um ambiente de CSCL enquanto as equipes de aprendizes trabalham e que o professor interage, de modo indireto, post hoc com os alunos e talvez apenas virtualmente. Na literatura em Educação Matemática, para o ensino com TD que possui essas especificidades, há pouca direção teórica para a elaboração de tarefas. Contribuindo a preencher esta lacuna, Powell e Alqahtani (2015) teorizam princípios metodológicos para elaborar tarefas para ambientes de CSCL e, principalmente, para compreender como as tarefas auxiliam uma equipe colaborativa de aprendizes a ser protagonista da sua aprendizagem. Os autores propõem alguns princípios metodológicos para a elaboração de tarefas:

1. Construção: fornecer uma construção de uma figura, instruções para a sua construção ou convite para a construção de uma figura com caraterísticas específicas.

2. Interação: convidar a equipe colaborativa de aprendizes a arrastar os pontos bases da figura e discutir entre si o que percebem sobre o comportamento, as relações entre objetos e as relações entre relações da figura.

3. Significado matemático: convidar os aprendizes a discutir os significados matemáticos de suas próprias percepções sobre o comportamento da figura.

4. Questionamento: convidar os aprendizes a propor questões sobre o que eles percebem ou significam matematicamente ou sobre a própria consequência disso.

5. Engajamento: para manter o envolvimento dos aprendizes, (1) fornecer pistas que sugerem aspectos relacionados à tarefa para discutir ou explicitar afirmações para revelar o que percebem, entendem ou se questionam matematicamente sobre o comportamento da figura e (2) sugerir desafios que aprofundem conhecimentos matemáticos envolvidos na tarefa. A princípio, as pistas e os desafios estão ocultos e podem ser revelados, clicando em um quadro de seleção no ambiente VMTwG, em particular, na janela geométrica de trabalho do software GeoGebra.

Zetetiké, Campinas, SP, v.24, n.2, maio/ago.2016, p.191-207

ISSN 2176-1744 
http://periodicos.sbu.unicamp.br/ojs/index.php/zetetike

6. Linguagem matemática: fornecer a linguagem matemática formal que corresponde ao que a equipe colaborativa de aprendizes está potencialmente sujeita a perceber e a discutir.

7. Feedback: responder em forma escrita ao trabalho de cada equipe colaborativa de aprendizes com a ideia de comentar sobre o que a equipe fez e não sobre o que não fez, convidá-la a revisitar algo, generalizar uma relação, justificar uma conjectura, propor novos desafios ou formular uma pergunta no formato "O que acontece se?". (pp. 87-88)

Esses princípios metodológicos para a elaboração de tarefas abrangem três intencionalidades. Duas delas se referem ao ambiente colaborativo virtual: representação (como GeoGebra) e comunicação (como rede social e chat). A terceira intencionalidade se vincula às próprias tarefas matemáticas como veículos "para estimular a criatividade, para incentivar a colaboração e estudar as ideias emergentes dos alunos" (Powell et al., 2009, p. 167, tradução nossa). Adicionalmente, as tarefas são elaboradas para serem discutidas e resolvidas de forma colaborativa.

Em relação à colaboração, Powell e Lai (2009) definem a interação colaborativa como troca de ideias, em que uns desafiam as ideias dos outros, uns afetam as ideias dos outros, todos trabalhando em conjunto com o mesmo propósito. Sobre a colaboração em ambientes computacionais, Alagic e Alagic (2013) apresentam três critérios: igualdade de participação, interações naturais e síntese unificada do trabalho. A colaboração concebe que os integrantes podem se sentir à vontade, interagindo, com objetivos comuns, negociados pela equipe. Por exemplo, quando professores de Matemática trabalham em pequenas equipes, isso pode contribuir de maneira significativa na construção do conhecimento matemático. Ao perceberem essa contribuição, eles podem utilizar projetos similares e trabalhar com seus estudantes em sala de aula (Alagic \& Alagic, 2013).

Nesse mesmo sentido, Powell e Grisi-Dicker (2012) discutem como equipes de professores em serviço investigam colaborativamente problemas abertos de Matemática e refletem sobre os registros de seus discursos. Para eles, as interações vivenciadas no VMTwG permitem aos professores o avanço dos conhecimentos matemáticos, pedagógicos e tecnológicos, no que se refere às relações com o objeto matemático e às relações entre relações.

A teoria psicológica de Gattegno (1987) explora a ideia de objetos matemáticos e a noção de suas relações, abordando o fazer matemática baseado em processos mentais, apresentando dois componentes: o diálogo e a percepção.

\footnotetext{
Ninguém duvida que a Matemática se sustenta por si mesma e é o mais claro dos diálogos da mente consigo mesma. A Matemática é criada por matemáticos conversando primeiro consigo mesmo e entre si. ... Baseado na percepção de que relações podem ser percebidas facilmente como objetos, a dinâmica que liga os diferentes tipos de relações foi extraída da mente de matemáticos e considerada por si mesma. (pp. 13-14, tradução nossa)
}

O diálogo é algo que um indivíduo faz consigo mesmo e com os outros. Tem conteúdo composto de percepções de três possíveis elementos específicos: objetos, relações entre os 
http://periodicos.sbu.unicamp.br/ojs/index.php/zetetike

objetos e dinâmicas que ligam as relações. Nesse viés, Sfard (2000) teoriza que o discurso matemático e seus objetos são mutuamente constitutivos. Entendemos que essa dupla ação reflexiva - mutuamente constitutivos - inclui as relações entre os objetos e as dinâmicas que ligam essas relações. A mente humana tem a facilidade natural para perceber ou tomar consciência de objetos e relações que encontra e também para estabelecer relações que ligam relações.

Para que o diálogo sobre esses três elementos faça sentido à aprendizagem é importante que ele: tenha uma permanência com algum tipo de registro gráfico; seja uma ação responsável e comprometida; seja uma atividade espontânea, mas deliberada e sujeita a ser repensada, relida e revisada. Esses atributos do diálogo são necessários para que ele seja uma fonte para a aprendizagem e para a reflexão profunda.

A teoria de Gattegno nos ajuda a entender quais as ações dos aprendizes correspondem ao fazer matemática e o que temos que observar para percebê-los fazendo matemática. As justificativas teóricas são maneiras de raciocinar com base nas relações entre objetos matemáticos e nas relações entre relações, ao contrário de justificativas baseadas na percepção visual, como, por exemplo, o uso de medidas. As observações sobre as relações são aprofundadas com o uso da escrita, um veículo que pode resultar de uma produção colaborativa num cenário presencial ou online e tem implicações na aprendizagem matemática. Essas ideias formam a base teórica deste trabalho. Em seguida, apresentamos os procedimentos metodológicos da pesquisa discutida neste artigo.

\section{Aspectos metodológicos}

A investigação apresentada neste artigo foi desenvolvida no âmbito da formação continuada de professores que atuam nos anos finais do Ensino Fundamental e no Ensino Médio nos Estados Unidos (EUA). As atividades foram realizadas por meio de uma disciplina online, com quatro equipes de professores que interagiram colaborativamente no ambiente VMTwG, o qual apresenta uma versão do software GeoGebra multiusuária e síncrona. Esse ambiente virtual foi desenvolvido por Stahl e seus colaboradores (Stahl, 2009). O VMTwG permite acesso livre ao público e é fruto de um projeto financiado pela National Science Foundation (NSF) nos Estados Unidos (EUA) (Powell, 2014).

As atividades desenvolvidas no ambiente são norteadas pelo engajamento dos professores convidados para a participação na formação continuada. As interações entre os professores focam o avanço da compreensão de objetos matemáticos, as relações entre eles e as dinâmicas criadas entre as diferentes relações matemáticas. As relações são geradas pela discussão de tarefas matemáticas de forma colaborativa (Powell \& Alqahtani, 2015).

No âmbito de nossa investigação, as tarefas foram previamente elaboradas e discutidas pelos professores que organizaram a disciplina (entre eles, os autores do artigo). Em nossa perspectiva, o conteúdo da disciplina tem como base a teoria do conhecimento tecnológico, pedagógico e do conteúdo, proposta por Mishra e Koehler (2006) e sofre 
http://periodicos.sbu.unicamp.br/ojs/index.php/zetetike

influência das ideias psicológicas de Gattegno (1987) sobre o fazer matemática (Powell, 2014). Nesta pesquisa, o conteúdo da disciplina contemplou 18 tópicos de geometria euclidiana plana, os quais foram discutidos com os professores em dois momentos distintos, cada um com duração de 15 semanas. No primeiro momento, as tarefas foram desenvolvidas de forma síncrona com o VMTwG e assincronamente com o Blackboard (similar ao Moodle). Enquanto, no segundo momento, usando as mesmas TD, acompanhamos o trabalho que os professores desenvolveram com seus estudantes, o que foi realizado pelos professores em sala de aula presencial e com o uso do VMTwG.

Neste artigo, apresentamos dois episódios sobre o primeiro momento. No âmbito geral, 14 professores participaram da formação continuada, constituindo quatro equipes (A, B, C e D). Especificamente, os referidos episódios derivam da discussão e da resolução, de forma colaborativa, de uma tarefa relativa ao tópico 14.04 - "Exploração de quadriláteros por meio de suas bissetrizes" -, pela Equipe D, composta por três professores, nomeados por Mihaela, Kelly e Tomas ${ }^{6}$ (Figura 1).

A maioria desses professores atua em escolas urbanas, com estudantes de famílias economicamente carentes e a minoria desses professores não possuía experiência com SGD, em particular, com o SketchPad, o Cabri ou mesmo o GeoGebra. Outro fator que justifica o trabalho com a geometria é que os referidos professores não possuem um conhecimento profundo da geometria euclidiana plana e isso tem implicações em como trabalham a disciplina na escola.

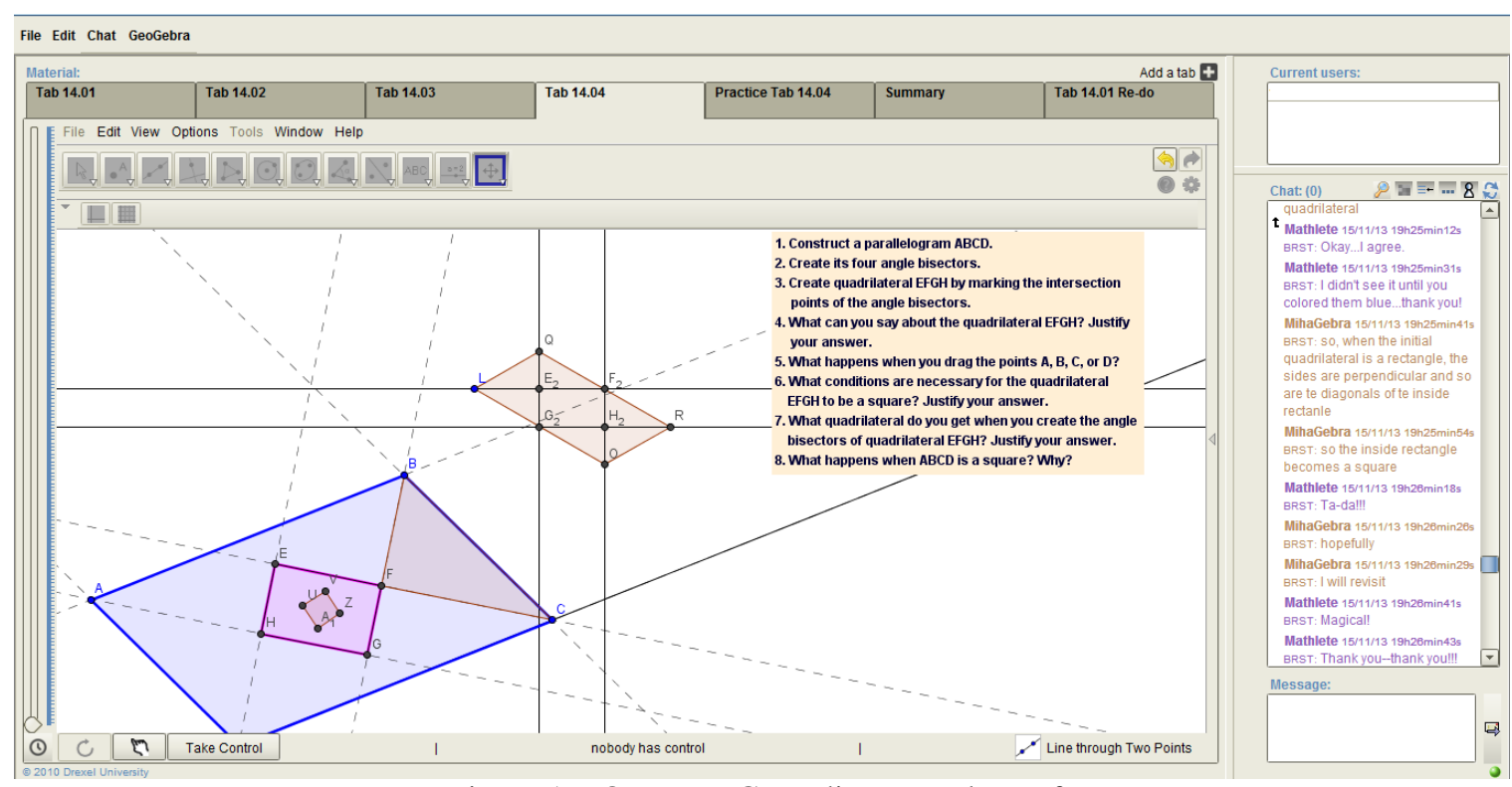

Figura 1 - O VMTwG e a discussão da tarefa

Fonte: VMTwG, retirado de http://vmt.mathforum.org/VMTLobby/commons/index.jsp.

\footnotetext{
${ }^{6}$ Temos a autorização para usar os nomes dos professores e também a discussão sobre as tarefas produzidas por eles.
} 
http://periodicos.sbu.unicamp.br/ojs/index.php/zetetike

Sendo assim, por meio dos dois episódios selecionados, buscamos mostrar como a equipe estabeleceu as justificativas geométricas colaborativamente entre os três participantes. As justificativas divergiram dos trabalhos de Chirstou et al. (2014) e de Amaral (2011). No primeiro, as relações foram realizadas individualmente e, no segundo, as interações foram construídas entre os professores e os pesquisadores. Em nossa análise, evidenciamos como as construções geométricas foram realizadas pelos professores, ao manipular ou arrastar pontos, e como eles, elaborando conjecturas e justificativas geométricas, nas quais buscaram a solução da tarefa sem o uso de ferramentas de medidas, perceberam as relações geométricas entre os objetos construídos e as relações entre relações.

$\mathrm{Na}$ apresentação e na discussão dos dados, contemplamos os itens da tarefa, as conjecturas, as justificativas escritas e os processos de validação manifestados pelos professores em relação aos objetos geométricos. Buscamos enfocar a colaboração como aspecto fundamental na construção de justificativas teóricas da tarefa proposta.

\section{Apresentação e discussão dos dados}

Em consonância com os procedimentos metodológicos, situamos a apresentação dos dados. Os dados se referem ao tópico 14.04. Entendemos ser pertinente esclarecer que o tópico 14 foi constituído por quatro tarefas (ou abas) no VMTwG, a saber: tópico 14.01: discutir e desenvolver hierarquias de quadriláteros; tópico 14.02: construir um quadrilátero, conectar os pontos médios dos seus lados a fim de formar um novo quadrilátero; investigar, descrever e justificar suas conjecturas sobre essa figura; tópico 14.03: construir um quadrilátero e em seguida as suas bissetrizes, investigar essa figura, descrever e justificar as conjecturas sobre o que sempre e em alguns casos é verdadeiro sobre isso.

Neste artigo, especificamente, apresentamos e analisamos a tarefa relativa ao tópico 14.04 (que também aparece na Figura 1), dos quadriláteros e suas bissetrizes, proposta aos professores. A tarefa propunha o seguinte:

1. Construa um paralelogramo ABCD.

2. Trace as suas quatro bissetrizes.

3. Trace o quadrilátero EFGH, marcando os pontos de intersecção das bissetrizes.

4. O que você pode dizer sobre o quadrilátero EFGH? Justifique sua resposta.

5. O que acontece quando você arrasta os pontos $\mathrm{A}, \mathrm{B}, \mathrm{C}$ ou $\mathrm{D}$ ?

6. Quais condições são necessárias para que o quadrilátero EFGH seja um quadrado? Justifique sua resposta.

7. Qual quadrilátero se forma, quando você traça as bissetrizes do quadrilátero EFGH? Justifique sua resposta.

8. O que acontece quando ABCD é um quadrado? Por quê?

(Tarefa traduzida da Língua Inglesa pelos autores).

Os professores discutiram e resolveram, de forma colaborativa, a tarefa em partes (itens de 1 a 8) no ambiente VMTwG. Por meio de dois episódios, apresentamos as 
http://periodicos.sbu.unicamp.br/ojs/index.php/zetetike

argumentações e as justificativas geométricas dos professores, na forma cronológica em que eles discutiram e resolveram a tarefa. Os episódios são constituídos pelas linhas numeradas do chat de discussão da tarefa. A discussão nos episódios foi traduzida da Língua Inglesa pelos autores. No Episódio 1, a discussão se refere aos primeiros cinco itens da tarefa, e no Episódio 2, a discussão se refere aos demais itens. A seguir, apresentamos o Episódio 1 e, $a$ posteriori, a nossa discussão sobre ele.

Antes do Episódio 1, os membros da Equipe D construíram um paralelogramo ABCD; traçaram as suas quatro bissetrizes e marcaram os pontos de intersecção das mesmas E, F, G e H (veja Figura 2). Agora, os professores estão conjecturando sobre o objeto geométrico, pensando o que as bissetrizes do paralelogramo $\mathrm{ABCD}$ formam e construindo uma justificativa.

\section{Episódio 1}

554 Kelly Tomas e eu estávamos tentando fazer a figura descrita em 14.04.

555 Mihaela Então as bissetrizes formam um retângulo.

556 Mihaela Esta é a conjectura?

557 Kelly Sim, esta foi a conjectura.

558 Kelly ângulos opostos são suplementares.

559 Mihaela Você provou isso?

560 Kelly Mas eu não havia pensado sobre isso realmente.

561 Mihaela Sim. Eu concordo.

Alguém pode pintar os pontos do triângulo que prove isso, digo, pintando cada respectivo

562 Mihaela triângulo com uma cor diferente?

563 Mihaela Nós estamos procurando um triângulo retângulo.

564 Mihaela Eu posso?

565 Kelly Isso seria ótimo!

566 Mihaela Desculpa, não dá.

567 Kelly O que você quer dizer? Eu penso que isso está perfeito!

568 Mihaela Eu fui enganada por esta configuração particular.

569 Kelly Você dividiu dois ângulos suplementares...

570 Mihaela Na realidade, isso funciona.

571 Mihaela Os ângulos consecutivos em um paralelogramo são suplementares.

No triângulo BCF, o ângulo FBC é a metade do ângulo B do paralelogramo, e o ângulo BFC é

572 Mihaela a metade do ângulo $\mathrm{C}$ do paralelogramo.

573 Mihaela Portanto a soma $\mathrm{m}(<\mathrm{FBC})+\mathrm{m}(<\mathrm{FCB})=90$.

574 Kelly Concordo!

575 Mihaela Ok, então as partes de 1 a 5 estão prontas.

576 Mihaela 1 a 5 estão prontas.

577 Mihaela Para a parte 6, quem quer investigar?

Percebemos a liderança de Mihaela, ao questionar os demais membros da Equipe D se a conjectura encontrada ao traçar as bissetrizes do paralelogramo culminaria na formação de um retângulo. Tomas não se pronuncia, mas Kelly afirma que essa é a conjectura. Os professores passam a buscar as relações geométricas para justificar tal conjectura e, em seguida, a sua validação. 
http://periodicos.sbu.unicamp.br/ojs/index.php/zetetike

Mihaela questiona Kelly se ela havia provado a conjectura. A partir disso, Mihaela usa os recursos do software GeoGebra para representar triângulos de diferentes cores, como aponta na linha 562: "Alguém pode pintar os pontos do triângulo que prove isso, digo, pintando cada respectivo triângulo com uma cor diferente?" A Figura 2 apresenta as representações dos triângulos realizadas por Mihaela.

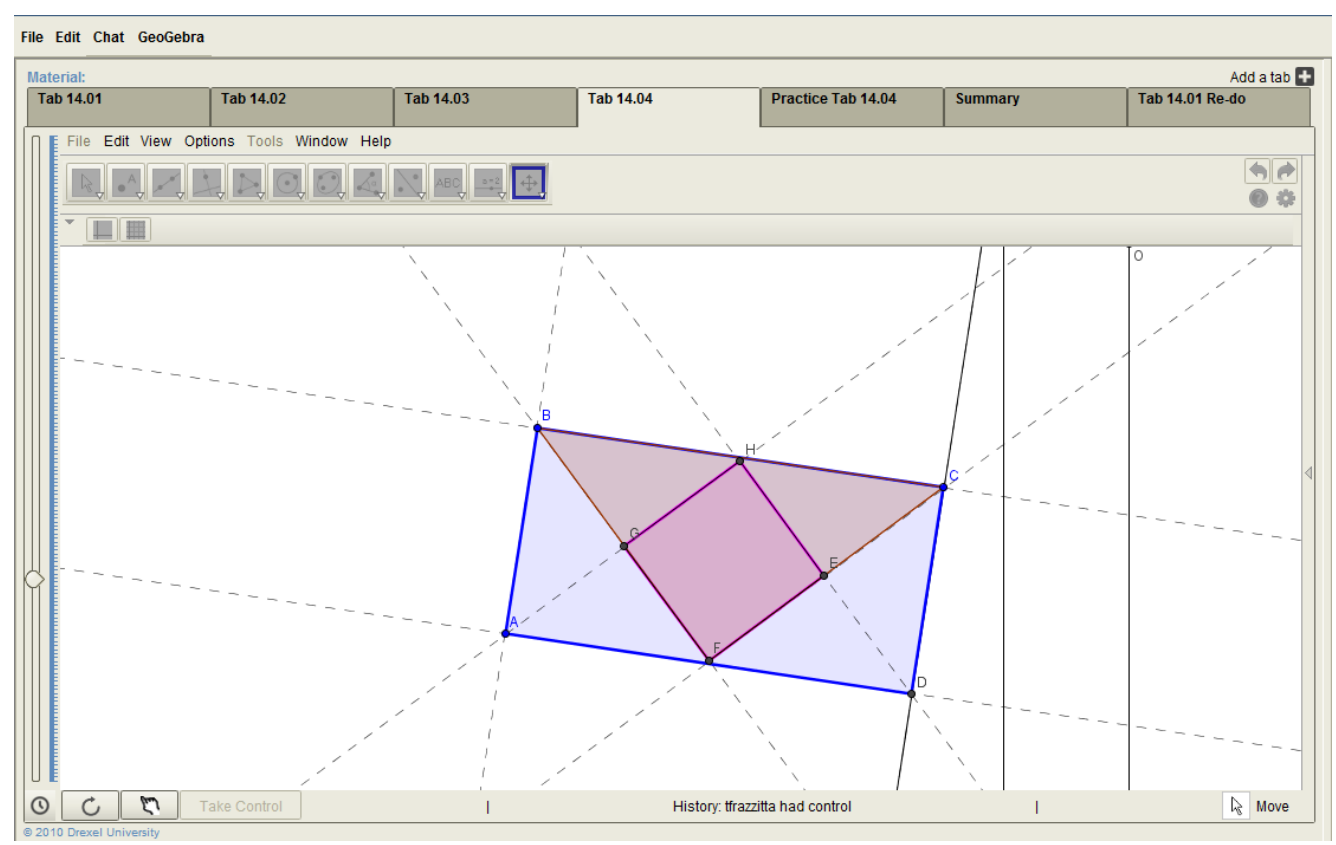

Figura 2 - Elaborando conjecturas

Fonte: VMTwG, retirado de http://vmt.mathforum.org/VMTLobby/commons/index.jsp.

Mihaela argumenta que os ângulos consecutivos de um paralelogramo são suplementares e, por meio dos seguintes argumentos: "No triângulo $B C F$, o ângulo $F B C$ é a metade do ângulo $B$ do paralelogramo e o ângulo BFC é a metade do ângulo $C$ do paralelogramo" (linha 572) e "Portanto a soma $m(<F B C)+m(<F C B)=90$ " (linha 573) justifica porque a figura formada é um retângulo. Os argumentos de Mihaela são aceitos por Kelly.

A partir disso, no Episódio 2, os integrantes da equipe passam a discutir os itens de 6 a 8 da tarefa e realizam as justificativas geométricas.

\section{Episódio 2}

714 Mihaela Mas eu penso que nós devemos discutir agora os tópicos de 6 a 8.

716 Kelly Eu adoraria. Eu quero ver como provar que é isso um quadrado. [...]

723 Mihaela Eu tentarei... Eu posso estar errada, eu preciso seu feedback. [...]

732 Mihaela Eu recriei a construção.

733 Mihaela Nós conhecemos a partir dos tópicos 4 e 5 que EFGH é um retângulo.

734 Mihaela Nós queremos saber quando ele se torna um quadrado.

735 Mihaela Para ser um quadrado, o retângulo deve ter suas diagonais perpendiculares.

736 Mihaela Você concorda? 
http://periodicos.sbu.unicamp.br/ojs/index.php/zetetike

737 Tomas Sim.

738 Mihaela Então eu construí a diagonal EG.

739 Kelly Concordo. E entendo porque ele é um retângulo.

740 Mihaela E eu quero provar que ela é paralela ao lado AB.

741 Mihaela E em particular do segmento BJ.

742 Kelly Oh, sim.

743 Kelly Sim, eu concordo.

744 Mihaela Nós sabemos que EJ é paralelo a HB.

745 Mihaela EJ é paralelo à HB.

746 Kelly Você disse "BJ".

747 Mihaela Também os segmentos r e s são congruentes.

748 Kelly Isso está ficando louco.

749 Mihaela Talvez eu deva usar algum tempo para eu verificar.

Se eu construir uma reta perpendicular GF paralela à $\mathrm{B}$, eu tenho os paralelogramos KBGF e

750 Mihaela KBHE.

751 Kelly Não. Está ok. Eu realmente aprecio isso! Continue.

752 Kelly Até agora isso está realmente interessante.

753 Kelly Eu realmente gostei da ideia das diagonais perpendiculares para provar o quadrado.

758 Tomas Eu tenho somente 27 minutos no computador... A biblioteca fecha rapidamente.

759 Mihaela Então eu tenho alguns lados congruentes

760 Mihaela Lados opostos. [...]

762 Kelly Então nós devemos focar nos lados congruentes $\mathrm{C} 1$, r e s?

763 Mihaela Isso é uma longa história.

764 Mihaela Os triângulos que formam são congruentes.

765 Kelly Desculpa... continue... Eu não devo interromper você.

766 Kelly Eu vejo os triângulos congruentes.

767 Mihaela Eventualmente.

768 Mihaela Desculpa... Isso é realmente uma longa história.

O ponto de partida, as diagonais do retângulo são paralelas em relação aos lados do quadrilátero

769 Mihaela inicial.

Eu realmente aprecio o trabalho... Eu sei que isso é realmente é um desafio para explicar

770 Kelly digitando.

Então, quando o quadrilátero inicial é um retângulo, os lados são perpendiculares e então são as

771 Mihaela diagonais do retângulo de dentro.

772 Kelly Ok. Eu concordo.

773 Kelly Eu não vi isso antes de você colorir eles em azul... Obrigado.

774 Mihaela Então o retângulo de dentro se torna um quadrado.

775 Kelly Pronto!

777 Mihaela Eu espero. [...]

779 Mihaela Eu revisitarei. [...]

786 Mihaela Se nós aceitarmos estes resultados, então 7 pode ser provada por esta mesma construção.

787 Kelly Incrível!

788 Kelly Obrigado-- obrigado!!! [...]

794 Mihaela Nós ainda temos que investigar 8.

799 Tomas EFEG será um quadrado também. [...]

801 Mihaela Isso parece um ponto.

802 Mihaela A prova disso é fácil.

803 Mihaela E rápida.

804 Kelly Oooh! Eu gosto de fácil e rápido (em Matemática!!!).

805 Mihaela Isso também se refere para as diagonais do quadrado original.

Zetetiké, Campinas, SP, v.24, n.2, maio/ago.2016, p.191-207 
http://periodicos.sbu.unicamp.br/ojs/index.php/zetetike

806 Mihaela [Risos].

807 Mihaela Sim, eu também.

808 Tomas Haha. [...]

810 Mihaela As diagonais de um quadrado são parte das bissetrizes. [...]

Então os quatro pontos definidos pela intersecção de quaisquer duas bissetrizes devem ficar

813 Mihaela sobre ambas as diagonais.

814 Tomas Sim, você está certa.

815 Kelly Ahh.

816 Mihaela Além disso, os quatro pontos coincidem com a intersecção das diagonais.

817 Kelly Então as bissetrizes interceptam em um simples ponto.

818 Tomas Concordo.

819 Kelly Mihaela, eu estou muito feliz em estar em sua equipe!

820 Tomas Duas bissetrizes criam a mesma reta.

821 Mihaela Sim.

822 Mihaela Ok, então todos concordamos.

Nesse episódio, novamente, Mihaela toma a iniciativa e solicita o feedback dos demais integrantes do Equipe D (linha 723). Entendemos que tal atitude configura um movimento de colaboração entre os participantes. Para explicitar as relações geométricas em relação aos itens 6 a 8, Mihaela faz uma construção paralela na aba de Prática do tópico 14.04, como mostra a Figura 3.

Mihaela e Kelly discutem a formação do quadrado (item 7) e o porquê tal figura geométrica é um quadrado (itens 6 e 8). As principais relações geométricas explicitadas por Mihaela se referem às diagonais do retângulo EFGH: "O ponto de partida, as diagonais do retângulo são paralelas em relação aos lados do quadrilátero inicial" (linha 769). A partir dessa relação, Mihaela apresenta as características do quadrado: "Então, quando o quadrilátero inicial é um retângulo, os lados são perpendiculares e então são as diagonais do retângulo de dentro" (linha 771). Essa relação geométrica é percebida por Kelly, quando Mihaela colore os segmentos de azul (Figura 3). Diante disso, Mihaela conclui: "Então o retângulo de dentro se torna um quadrado" (linha 774) e Kelly concorda.

Em seguida, os professores passam a investigar o item 8, que toma o quadrilátero inicial ABCD como um quadrado. Também nesse caso, Mihaela usa a mesma relação geométrica para justificar, já que "As diagonais de um quadrado são parte das bissetrizes" (linha 810). As justificativas geométricas de Mihaela "Então os quatro pontos definidos pela intersecção de quaisquer duas bissetrizes devem ficar sobre ambas as diagonais" (linha 813) e "Além disso, os quatro pontos coincidem com a intersecção das diagonais" (linha 816) foram aceitas por Tomas e Kelly. Kelly, também, complementa afirmando que "Então as bissetrizes interceptam em um simples ponto" (linha 817). Sendo assim, os professores avaliam as justificativas geométricas e finalizam a discussão da tarefa. 
http://periodicos.sbu.unicamp.br/ojs/index.php/zetetike

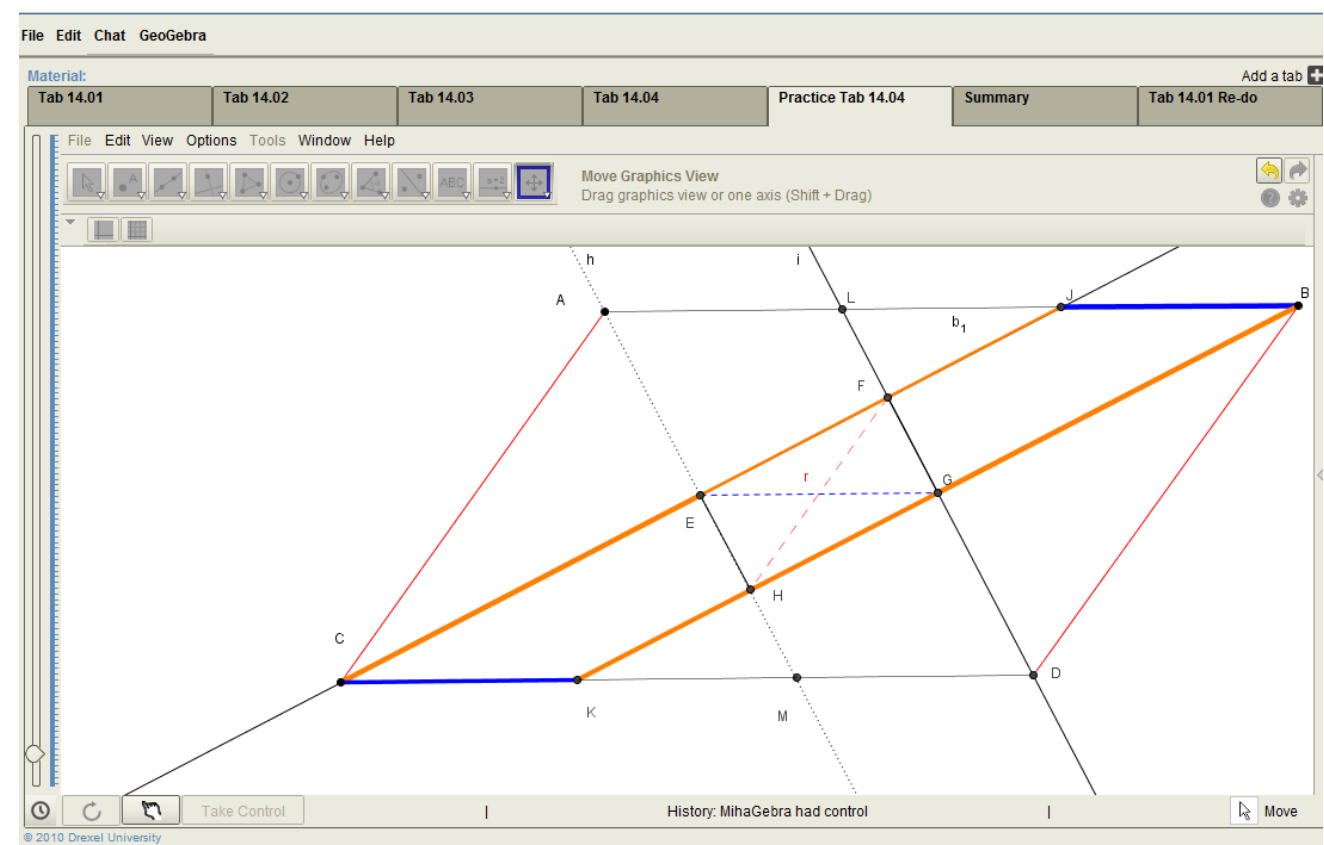

Figura 3 - Construindo relações geométricas

Fonte: VMTwG, retirado de http://vmt.mathforum.org/VMTLobby/commons/index.jsp

\section{Discussão dos resultados}

Nesta seção, em referência a uma tarefa específica no ambiente virtual, VMTwG, apresentamos como três professores de Matemática, colaborativamente, realizaram construções geométricas e justificativas teóricas, discutindo três aspectos que constituem os resultados deste trabalho: justificativas, colaboração e características da tarefa.

Tomando como base as construções em geometria dinâmica, evidenciamos como uma equipe de três professores do Ensino Fundamental elaboraram suas conjecturas; explicitaram suas relações baseadas nas propriedades dos objetos geométricos, de relações entre os objetos, de relações entre relações (Gattegno, 1987); e construíram justificativas teóricas. As justificativas dos professores, ao manipular e construir objetos em geometria dinâmica, evidenciaram as propriedades ("os ângulos consecutivos em um paralelogramo são suplementares"), as relações entre os objetos ("Para ser um quadrado, o retângulo deve ter suas diagonais perpendiculares") e as relações entre relações ("As diagonais de um quadrado são parte das bissetrizes. ... Então os quatro pontos definidos pela intersecção de quaisquer duas bissetrizes devem ficar sobre ambas as diagonais").

Enfatizamos que o processo de justificativas geométricas ocorreu de forma colaborativa (Alagic \& Alagic, 2013), pois os três critérios para tal foram atendidos: igualdade de participação, pois os professores participantes possuíam as mesmas possibilidades de resolução e discussão, sendo professores da Educação Básica; interações naturais, visto que os professores trabalharam na elaboração de justificativas geométricas para a tarefa em questão em comum acordo; e a síntese unificada do trabalho, em que os 
http://periodicos.sbu.unicamp.br/ojs/index.php/zetetike

professores discutiram de forma colaborativa e discursiva sobre cada tópico da tarefa, produzindo sínteses parciais e conclusivas (Mihaela, “Ok, então todos concordamos").

Outro aspecto a ser discutido é a importância de tarefas dessa natureza, investigativa e exploratória, para a construção de conhecimentos do professor de Matemática e para o posterior trabalho com seus estudantes, seja no aprimoramento das estratégias didáticas, como no conhecimento para o ensino na Educação Básica. Como mencionamos na introdução, as justificativas geométricas elaboradas pelos professores é um dos movimentos que os professores podem adotar na trabalho com os estudantes.

Para discutir esse movimento em um ambiente CSCL, tomamos como referência os princípios metodológicos elaborados por Powell e Alqahtani (2015). Em contraste às investigações anteriores com a mesma tarefa, as de Christou et al. (2004) e de Amaral (2011), em vez do uso de ferramentas de medida, mostramos o movimento de discussão e de resolução de uma tarefa, o qual promoveu a extensão das capacidades de argumentações e de justificativas particulares de professores em um ambiente CSCL, mobilizando a prova, tomando como base as relações geométricas.

Sugerimos que o conjunto de princípios metodológicos elaborados por Powell e Alqahtani (2015) influenciou nas justificativas dos professores para a resolução e a discussão da tarefa do paralelogramo e suas bissetrizes, tomando como sustentação as relações geométricas. Para evidenciar isso, discutimos as sete características em consonância com os episódios apresentados.

Os participantes foram convidados a fazer a construção de um paralelogramo (itens 1, 2 e 3 da tarefa) e, a partir daí, iniciaram a interação e a produção de significado matemático. Por exemplo, as questões "4. O que você pode dizer sobre o quadrilátero EFGH? Justifique sua resposta" e "5. O que acontece quando você arrasta os pontos A, B, C ou $D$ ?" permitiram aos professores a elaboração de conjecturas matemáticas, com base nas propriedades das figuras geométricas, conforme já abordamos na discussão dos dados, com auxílio da Figura 2.

Também, a natureza da tarefa convidava os professores para propor questionamentos a partir das conjecturas, como por exemplo: "Nós conhecemos a partir dos tópicos 4 e 5 que EFGH é um retângulo; Nós queremos saber quando ele se torna um quadrado. Para ser um quadrado, o retângulo deve ter suas diagonais perpendiculares. Você concorda?" (Mihaela, Episódio 2). Diante desse questionamento, a referida professora provocou o engajamento dos demais professores na discussão e a resolução dos tópicos que compunham a tarefa, como percebemos pelas respostas dos demais professores: "Tomas: "sim" e Kelly: "Concordo. E entendo porque ele é um retângulo".

Por último, acreditamos que a tarefa apresentou uma linguagem matemática que corresponde ao foco de ensino da equipe de professores em discussão. Assim como atendeu ao princípio metodológico de elaboração de questões no formato "O que acontece se?", em evidência no tópico: "8. O que acontece quando $A B C D$ é um quadrado? Por quê?", que 
http://periodicos.sbu.unicamp.br/ojs/index.php/zetetike

permitiu a resolução da tarefa por meio de relações geométricas, conforme abordamos na discussão dos dados, com o auxílio da Figura 3.

A partir da discussão dos resultados, elaboramos um diagrama (Figura 4) que contempla o movimento de formação continuada de professores de Matemática no VMTwG. Sinteticamente, o diagrama mostra que, por meio de um roteiro de tarefas, os professores produziram justificativas teóricas para as questões propostas na tarefa "Paralelogramo e suas bissetrizes". As justificativas foram baseadas nas propriedades dos objetos matemáticos, nas relações entre objetos e nas relações entre relações. O processo de discussão e resolução das tarefas ocorreu colaborativamente entre os professores.

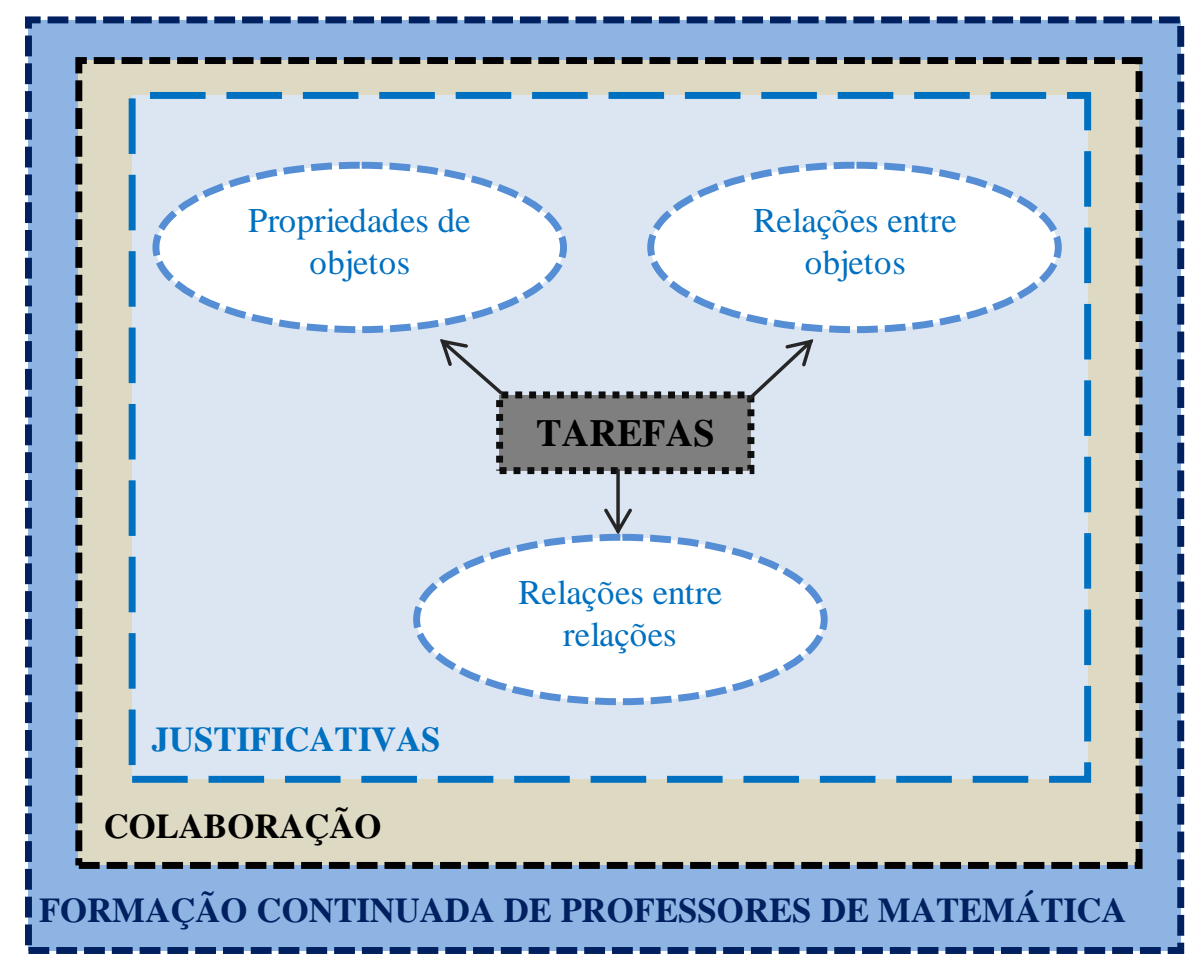

Figura 4 - Diagrama da discussão dos resultados

Fonte: os autores.

Consideramos que este trabalho pode dialogar com a formação de professores de Matemática. No próximo tópico, apontamos contribuições dos resultados de nossa pesquisa para a formação de professores, tanto inicial como continuada, especialmente, em ambientes virtuais de aprendizagem.

\section{Contribuições para a formação de professores de matemática}

Neste artigo, evidenciamos que os resultados desta pesquisa podem contribuir para a prática docente e, consequentemente, na Matemática a ser ensinada para os estudantes em sala de aula. Afirmamos isso, pois esse curso de formação promoveu o encontro, a colaboração e o compartilhamento dos conhecimentos mobilizados por professores em exercício. 
http://periodicos.sbu.unicamp.br/ojs/index.php/zetetike

Os conhecimentos dos professores que destacamos se vinculam ao conjunto de conhecimentos do conteúdo matemático, pedagógico e tecnológico proposto por Mishra e Koehler (2006). Em outras palavras, acreditamos que o conjunto de tarefas elaborado no tópico 14, compreendido de tarefas abertas e específicas, pôde evidenciar esse corpo de conhecimentos. Em Powell (2014), há uma discussão sobre os referidos conhecimentos.

Como já explicitamos, a discussão matemática foi a que se sobressaiu, pois nosso foco de investigação procurou mostrar o movimento intrínseco de professores desde o processo de exploração até a justificação teórica, sem o uso de medidas. A identificação desse movimento foi possível, entre outros aspectos, pela elaboração de tarefas (Powell \& Alqahtani, 2015), assim como pelas tarefas que antecederam o tópico 14.04, as quais contribuíram para o diálogo dos conhecimentos matemáticos revelados pelos professores em formação continuada.

A elaboração das tarefas é um componente fundamental do conhecimento pedagógico do professor (Powell \& Alqahtani, 2015; Powell, 2014). Afirmamos isso, pois entendemos que a vivência de tarefas investigativas dessa natureza é um aspecto pedagógico a ser contemplado nos cursos de formação de professores, seja no âmbito da Licenciatura em Matemática, como em ações de formação continuada.

Também salientamos a presença do conhecimento tecnológico ao usar o ambiente VMTwG, em particular, no trabalho com o software GeoGebra. O conhecimento tecnológico permitiu aos professores a elaboração de conjecturas, a realização de testes e a justificativa geométrica. Assim como a colaboração se potencializou pelo próprio trabalho no ambiente VMTwG. Ainda, sugerimos a realização de pesquisas sobre como professores podem aprimorar as construções geométricas e realizar justificativas teóricas, usando TD em sala de aula.

\section{Referências}

Alagic, G., \& Alagic, M. (2013). Collaborative mathematics learning in online environments. In D. Martinovic, V. Freiman, \& Z. Karadag (Eds.), Visual Mathematics and Cyberlearning (Vol. 1, pp. 23-48). New York: Springer.

Amaral, R. B. (2011). Argumentação matemática colaborativa em um ambiente online. Acta Scientiae, 13(1), 55-70. Retirado em 12 de novembro de 2013, de http://www.periodicos.ulbra.br/index.php/acta/article/view/23/20.

Arzarello, F., Olivero, F., Paola, D., \& Robutti, O. (2002). A cognitive analysis of dragging practises in Cabri environments. International Reviews on Mathematical Education (ZDM), 34(3), 66-72.

Christou, C., Mousoulides, N., Pittalis, M., \& Pitta-Pantazi, D. (2004). Proofs through exploration in dynamic geometry environment. International Journal of Science and Mathematics Education, 2(3), 339-352.

Gattegno, C. (1987). The science of education: Part 1: Theoretical considerations. New York: Educational Solutions. 
http://periodicos.sbu.unicamp.br/ojs/index.php/zetetike

Hölzl, R. (2001). Using dynamic geometry software to add contrast to geometric situations A case study. International Journal of Computers for Mathematical Learning, 6(1), 63-86.

Mishra, P., \& Koehler, M. J. (2006). Technological pedagogical content knowledge: A framework for teacher knowledge. Teachers College Record, 108(6), 1017-1054.

Öner, D. (2008). Supporting students' participation in authentic proof activities in computer supported collaborative learning (CSCL) environments. Computer-Supported Collaborative Learning, 3, 343-359.

Powell, A. B. (2014). Construção colaborativa do conhecimento tecnológico, pedagógico e do conteúdo de professores de matemática. Boletim GEPEM, 64, 88-103.

Powell, A. B., \& Alqahtani, M. M. (2015). Tasks and meta-tasks to promote productive mathematical discourse in collaborative digital environments. In I. Sahin, S, A. Kiray, \& S. Alan (Eds.), Proceedings of the International Conference on Education in Mathematics, Science \& Technology (pp. 84-94). Antalya, Turkey.

Powell, A. B., Borge, I. C., Floriti, G. I., Kondratieva, M., Koublanova, E., \& Sukthankar, N. (2009). Challenging tasks and mathematics learning. In E. J. Barbeau, \& P. J. Taylor (Eds.), Challenging mathematics in and beyond the classroom: The 16th ICMI study (pp. 133-170). New York: Springer.

Powell, A. B., \& Grisi-Dicker, L. (2012). Toward collaborative, discourse-focused learning with dynamic geometry environment. In Proceedings of the 12th International Congress on Mathematical Education (pp. 1-10). Seoul, Korea: ICME.

Powell, A. B. \& Lai, F. F. (2009) Inscriptions, mathematical ideas, and reasoning in VMT. In G. Stahl (Ed.), Studying virtual math teams. (ch 13, pp. 237-259). New York, NY: Springer.

Sfard, A. (2000). Symbolizing mathematical reality into being. Or how mathematical discourse and mathematical objects create each other. In P. Cobb, E. Yackel, \& K. McClain (Eds.), Symbolizing and communicating in mathematics classrooms: Perspectives on discourse, tools, and instructional design (pp. 37-98). Mahwah, NJ: Lawrence Erlbaum Associates.

Silverman, J. (2011). Supporting the development of mathematical knowledge for teaching through online asynchronous collaboration. The Journal of Computers in Mathematics and Science Teaching, 30, 61-78.

Stahl, G. (Ed.). (2009). Studying virtual math teams. New York: Springer.

Stahl, G., Koschmann, T., \& Suthers, D. (2008). Aprendizagem colaborativa com suporte computacional: Uma perspectiva histórica. Boletim GEPEM, 53, 11-42.

Submetido em: 17/12/2015

Aceito em: 18/04/2016 
http://periodicos.sbu.unicamp.br/ojs/index.php/zetetike 